\title{
Synchronous Primary Ovarian Mucinous Carcinoma and Endometrioid Endometrial Carcinoma : A Rare Case Report
}

\author{
Mohan Krishna Pasam ${ }^{1}$, Rajendiran $\mathrm{S}^{1 *}$, Susruthan $\mathrm{M}^{1}$ and Usha Rani ${ }^{2}$ \\ 'Department of pathology, Sri Ramachandra Medical college and Research Institute ,chennai(India) . \\ ${ }^{2}$ Department of obstetrics and gynaecology . Sri Ramachandra Medical College and Reasearch Institute, Chennai(India)
}

\section{ABSTRACT}

Introduction: Synchronous tumours of female genital tract are a rare . The most common organs involved are ovary and endometrium. Endometrioid carcinoma is the most common malignancy detected in majority of cases. Synchronous cancers with dissimilar histology are a very rare phenomenon. We report a case of 51 year old lady with synchronous primary mucinous carcinoma of ovary and primary endometrioid endometrial carcinoma .

Case Report: a 51 year old lady presented with post menopausal bleeding and foul smelling vaginal discharge of 1 month duration . Her CA125 was elevated. Magnetic resonance imaging showed increased endometrial thickness and a12.5x10x9.3 cm mass in the fundus of uterus suggestive of a leiomyoma along with and a $13.8 \times 11.5 \times 11 \mathrm{~cm}$ mass in the left adnexa. Endometrial aspiration done showed Complex Hyperplasia with Atypia. Total abdominal hysterctomy and bilateral salphingoophorectomy along with lymphnode sampling was performed. The adnexal mass was pT1a pN0 cM0 grade 2 invasive mucinous carcinoma. The endometrial lesion was pT1b pN0 cM0 FIGO grade 2 endometrioid endometrial adenocarcinoma.

Conclusion: Synchronous ovarian tumours of dissimilar histology is extremely rare and there are only a few reports of ovarian mucinous carcinoma with endometrioid endometrial carcinoma.

\section{Keywords: Synchronous, Ovarian Tumour, Endometrial Adenocarcinoma, Immunohistochemistry}

\section{Introduction}

Simultaneously identified tumours in female genital tract represent any of the three following possibilities . Primary endometrial tumour with ovarian metastasis . Primary ovarian tumour with metastasis to the endometrium . Synchronous primary ovarian and endometrial carcinoma. Synchronous primary tumours of female genital tract account for only $<2 \%$ of all gynaecological malignancies $[1,2]$. Ziano $\mathrm{R}$ et al reported that $5 \%$ cases of endometrial cancer have synchronous tumour in ovary and $10 \%$ cases of ovarian cancer show a synchronous tumour in the endometrium ${ }^{[3]}$. Scully et al proposed a clear pathological criteria for determining synchronous tumours which include histological dissimilarity, minimal invasion of endometrial tumour, presence of in situ carcinoma, absence of vascular space invasion of endometrial tumour , unilateral ovarian tumour involving parenchyma with no surface involvement, presence of ovarian endometriosis ${ }^{4}$ We report a 51 year old woman presented in our centre with post menopausal bleeding diagnosed to have a rare synchronous association of primary ovarian mucinous carcinoma and endometrioid endometrial carcinoma

\section{Case Report}

A 51 year old lady came with post menopausal bleeding for 1 month and foul smelling vaginal discharge for 1 week. Her Body Mass index was $28 \mathrm{~kg} / \mathrm{m}^{2}$ and is a known case of hypothyroid on treatment .She had undergone total knee replacement for osteoarthritis 3years back. Physical examination showed an abdominal mass corresponding to 18 to 20 weeks gestation. Magnetic resonance imaging was done which showed thickened endometrium of $57 \mathrm{~mm}$ along with a heterogenous conglomerate mass in the fundus of size $12.5 \times 10 \times 9.3 \mathrm{~cm}$ [fig.1] suggestive of a leiomyoma. A well circumscribed heterogenous and hyper intense adnexal mass of $13.8 \times 11.5 \times 11 \mathrm{~cm}$ was also seen[Fig.1]. Serology showed an elevated CA125. Pre operative endometrial aspiration was done which showed Complex Hyperplasia with Atypia. A staging laparotomy was done and total abdominal hysterectomy with bilateral salphingoophorectomy was sent for frozen section. The adnexal mass weighed $2.5 \mathrm{~kg}$ and measured $18 \times 16 \times 7 \mathrm{~cm}$ with a smooth grey white nodular surface. The cut curface exuded yellowish mucoid material and showed $80 \%$ solid areas and $20 \%$ cystic areas with papillary projections [fig:2]. The uterus showed friable lesion in the lower uterine segment and body and was infiltrating $>50 \%$ of the myometrium [fig:3].A grey white well circumscribed mass of size $13.5 \times 8 \times 7 \mathrm{~cm}$ seen in myometrium. Histopathology of the sections from the adnexal mass showed mucinous carcinoma of endocervical type which was invading the 
ovarian stroma[Fig.4].while the endometrium showed endometrioid type endometrial adenocarcinoma with myometrial invasion[Fig.5] . Further pelvic lymph node sampling was done and a final diagnosis of pT1a pN0 cM0 grade 2 invasive mucinous carcinoma of left ovary and pT1b pN0 cM0 FIGO grade 2 endometrioid endometrial adenocarcinoma was given. The grey myometrial mass showed features of a leiomyoma and opposite ovary showed endometriosis[Fig.6]. Immunohistochemistry done on both ovarian and endometrial tumours showed a positive nuclear staining of estrogen receptor in endometrial carcinoma ${ }^{[} \mathrm{Fig}$. 7] and was negative in ovarian mucinous carcinoma .

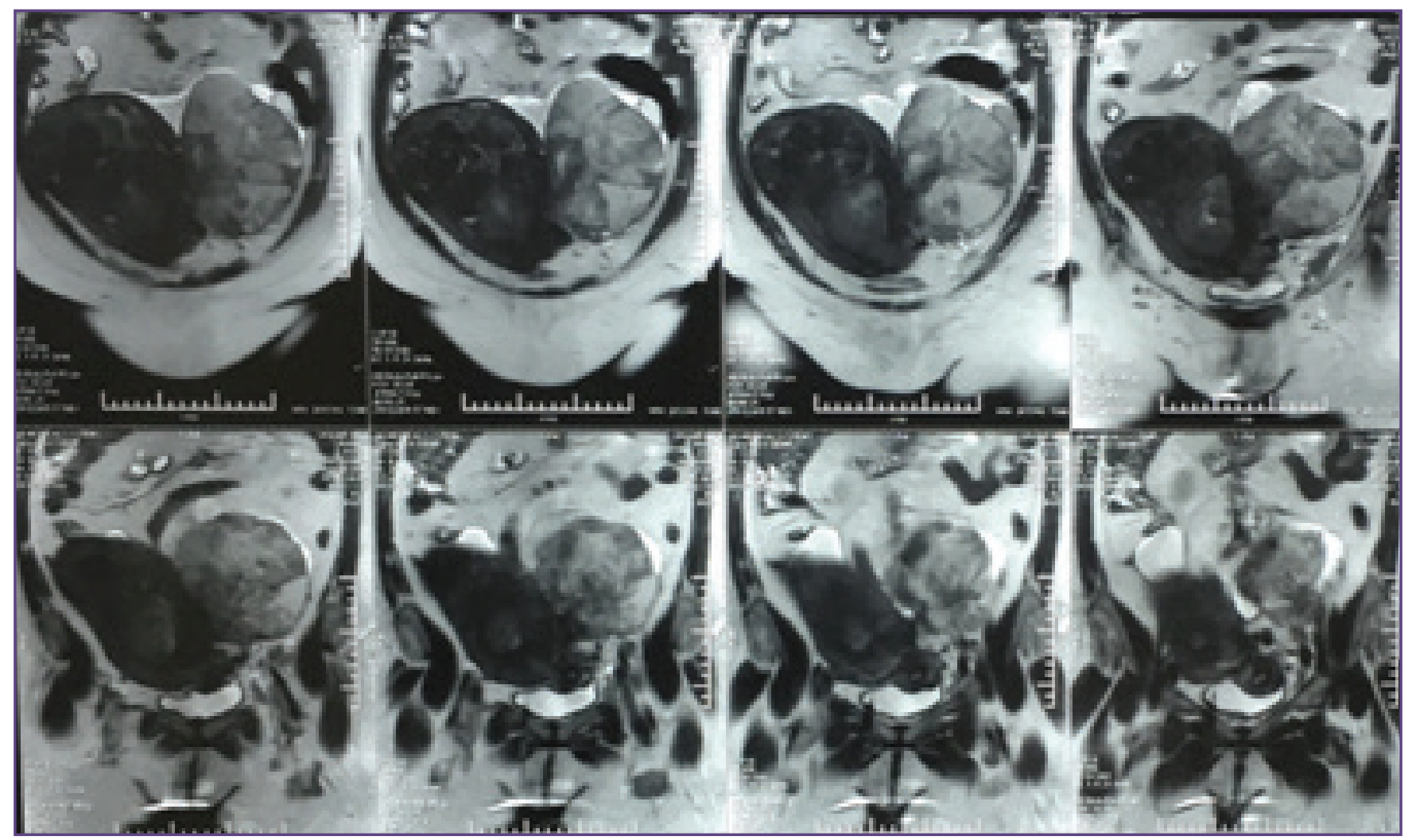

Fig. 1: MRI showing an adnexal and uterine mass.

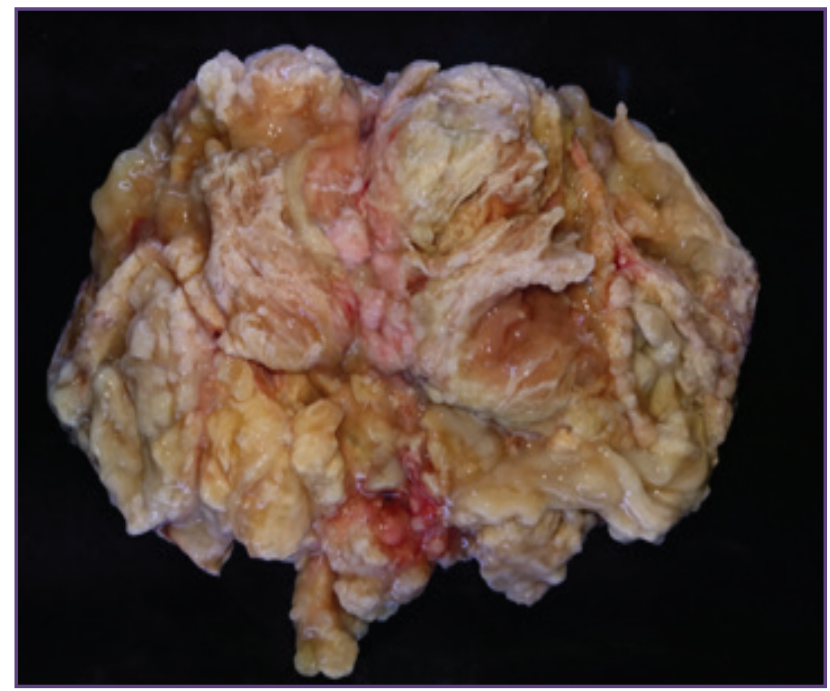

Fig. 2 : ovarian mass showing a multiloculated lesion with papillary architecture and mucin.

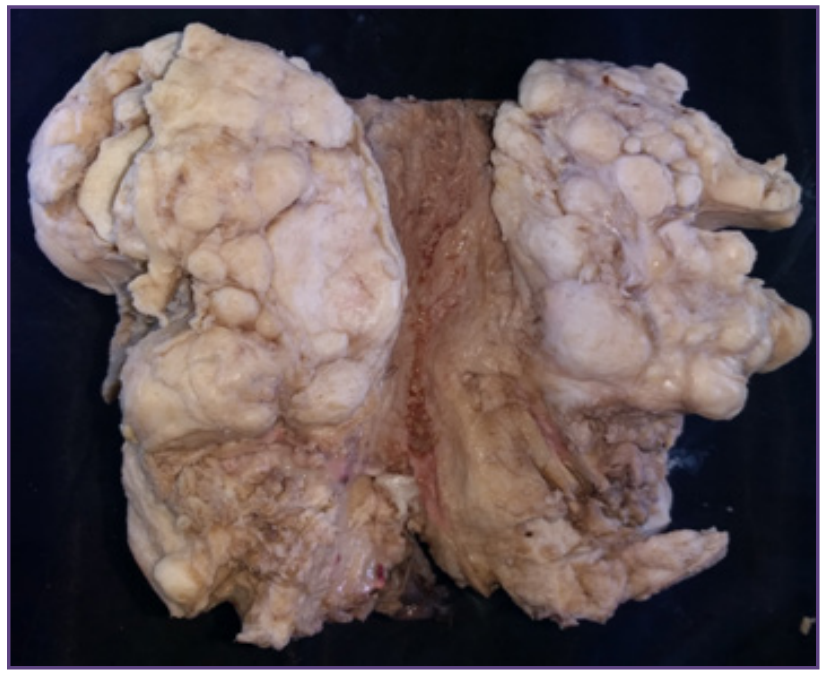

Fig. 3 : uterus with endometrial lesion and leiomyoma. 


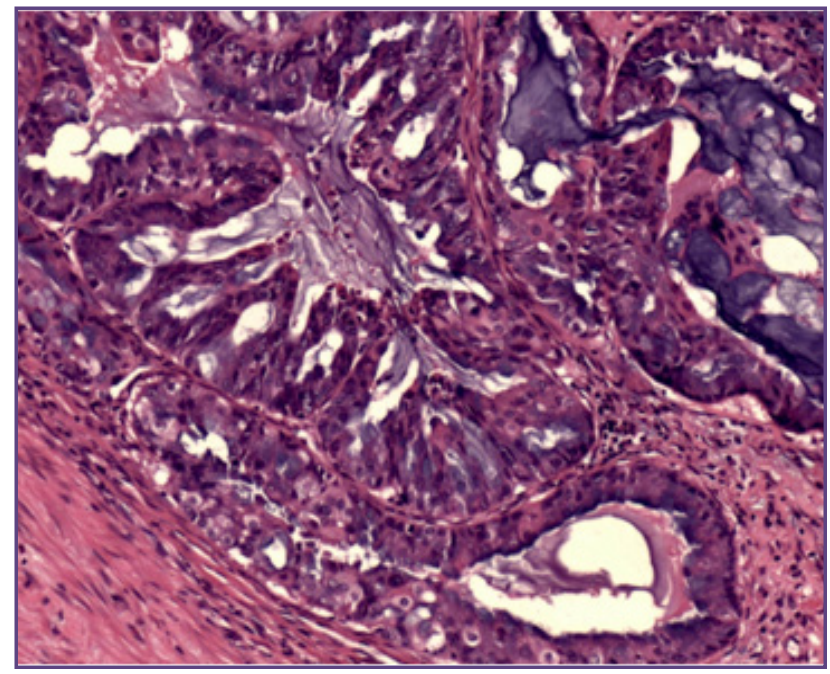

Fig. 4: H\&E , invasive mucinous carcinoma of ovary : 200x.

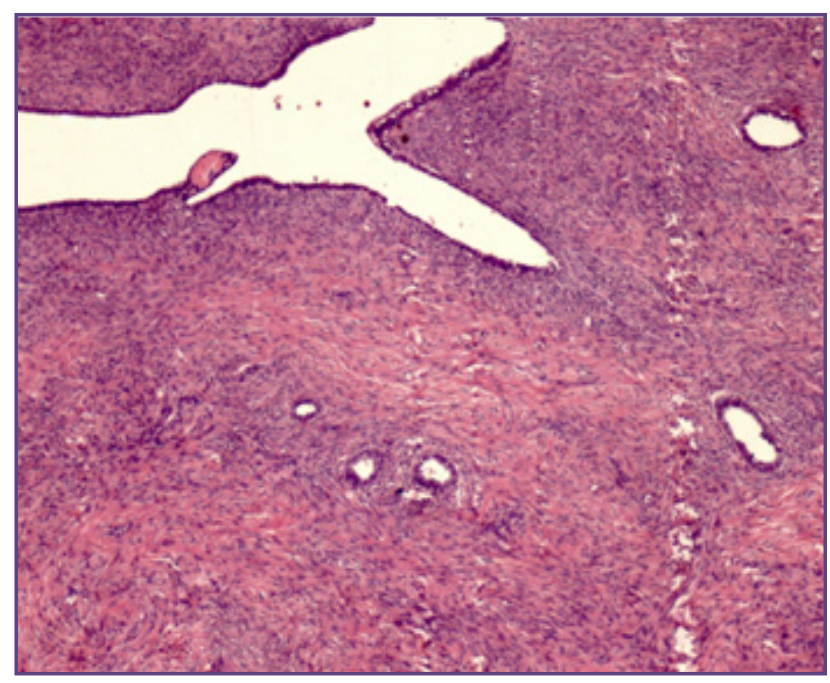

Fig. 6: H\&E : opposite ovary with endometriosis 40x.

\section{Discussion}

Our case fulfilled all the pathological criteria for a synchronous malignancy. In a study done by Y Chiang et al done on 23 cases of synchronous ovarian and endometrial tumours $63 \%$ showed similar histology $y^{[5]}$. A 10 year study done by Young Kuei Lim et al on 75 patients with synchronous tumours showed $73.9 \%$ being endometrioid endometrial cancers and $26.1 \%$ with tumours of different histological type ${ }^{[6]}$. Yuantaoliu et al reported 43 cases of synchronous tumours of which $30 \%$ showed dissimilar histology ${ }^{[7]}$.There are only a few reports of synchronous endometrial carcinoma with an ovarian carcinoma of mucinous morphology. Catharina et al reported 53 cases of synchronous endometrial and ovarian tumours of which

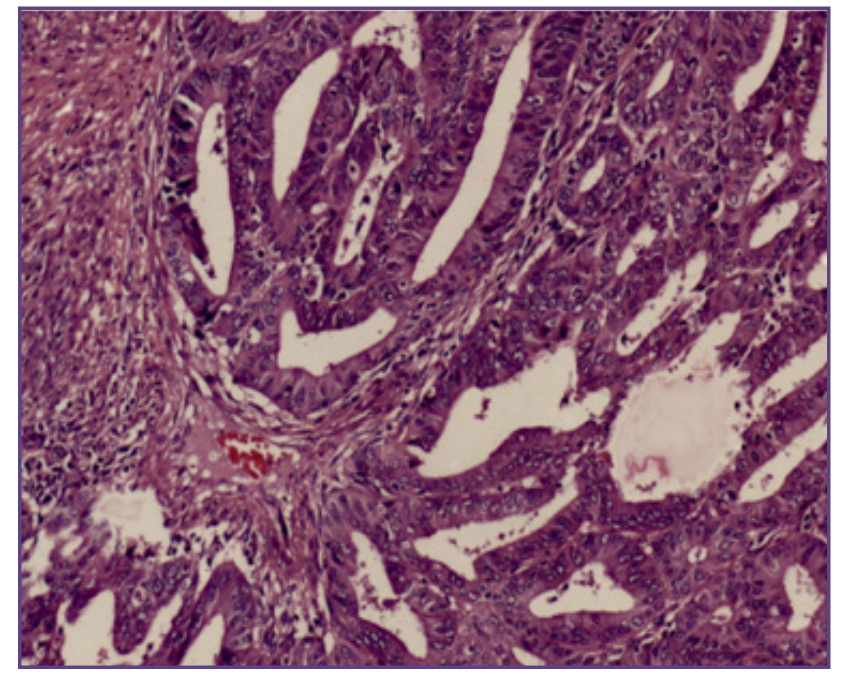

Fig. 5: H\&E endometrium showing invasive endometrioid adenocarcinoma : 100x.

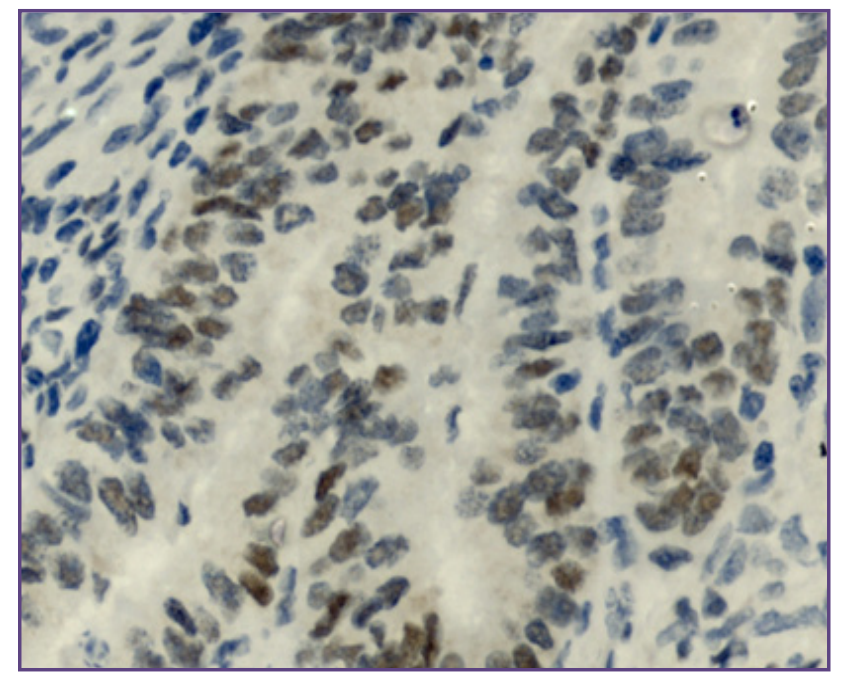

Fig. 7: immunohistochemistry positive for Estrogen receptor in endometrial carcinoma 400x

$75 \%$ were endometrioid carcinomas ${ }^{8}$. They reported 7 cases of ovarian mucinous carcinomas with endometrioid endometrial carcinomas in their study. Sylwia et al reported 10 cases of which 1 was ovarian mucinous carcinoma with endometrioid endometrial carcinoma ${ }^{[9]}$. Age of presentation in our case was 51 years which is correlating with previous studies ${ }^{[7] .}$

Presenting symptom of synchronous tumours is that of individual primary tumours ${ }^{[7]}$. In our case it was post menopausalbleeding. Soliman PT et al reported that 30 to $40 \%$ of patients with synchronous tumours were obese $\left(\mathrm{BMI}>30 \mathrm{~kg} / \mathrm{m}^{2}\right)^{[10]}$. Though obesity is a known risk factor for endometrial carcinoma, there is no specific data to 
support the association of obesity with synchronicity. Majority of patients with synchronous tumours show elevated CA125 levels. Yuantaoliu et al reported a mean CA125 value of $161.7 \mathrm{IU} / \mathrm{ml}$ in their study ${ }^{[7]}$. Endometriosis of ovaries is seen in majority of cases with synchronous tumours ${ }^{[3]}$. Though our case had endometriosis in the opposite ovary its association with mucinous ovarian carcinoma could not be explained.

Synchronous tumours clinically present at a lower stage at diagnosis when compared to the tumours of individual organs. Previous reports do suggest that majority of synchronous tumours present at stage $\mathrm{I}^{[4,7]}$. The treatment of synchronous endometrial and ovarian cancer depends on the pathological characteristics of each tumour ${ }^{[4,11]}$. Surgery with an adjuvant chemotherapy remains the main stay of treatment. However reports show that stage I and II ovarian tumours which are grade 1 and endometrial tumours which are grade 1 with only superficial myometrial invasion do not require chemotherapy . In our case both endometrial and ovarian tumours were stage I. Long term prognosis of synchronous tumours was found to be relatively good . The prognosis often depends on the stage of each tumour rather than the histological type. Studies have shown that 5 year survival rates are excellent. Zaino $\mathrm{R}$ et al reported $86 \% 5$ year survival and $80 \% 10$ year survival ${ }^{[3]}$. Another report has shown a mean survival of $84 \%{ }^{6}$. Yuantaoliu et al reported a mean survival of 109 months and rate of $93.5 \%$ in stage I and stageIItumours ${ }^{[7]}$.Presentation at an early stage may be attributed to the overall good prognosis .

Pathogenesis of synchronous ovarian and endometrial tumours is still unclear. Various theories like shared molecular receptors in different organs of female genital tract which respond to same carcinogen may hold good for tumours of similar histology . But This theory cannot explain the occurrence of tumours with dissimilar histology - Molecular study done by Soliman et al showed on $7 \%$ of cases with criteria suggestive of a Lynch syndrome ${ }^{[10]}$. Wether the synchronicity of tumours of different histology is incidental or due to a common molecular carcinogenesis is yet to be determined.

\section{Conclusion}

Primary ovarian carcinoma with a synchronous primary tumour in the endometrium though a rare phenomenon is occasionally encountered in gynecological practice. Majority of synchronous tumours in the ovary and endometrium are of endometrioidtype. The occurrence of tumours with dissimilar histology is a rare event.
There are very few reports of synchronous mucinous carcinoma ovary and endometroid endometrial carcinoma. This case is reported for its rarity, to emphasise the importance of thorough radiological evaluation of endometrium in all adnexal mass lesions and to emphasise the need of endometrial aspiration in suspicious cases. There is a dire need for extensive molecular analysis for determining the pathology of these synchronous tumours with dissimilar histology.

\section{Acknowledgements}

Dr Sandhya Sundaram, Head, Department of pathology, Sri Ramachandra Medical college, Chennai.

\section{Reference}

1. Tong SY, Lee YS, Park JS, Bae SN, Lee JM, Namkoong SE. Clinical analysis of synchronous primary neoplasms of the female reproductive tract. European Journal of Obstetrics \&Gynecology and Reproductive Biology. 2008 Jan 31;136(1):78-82.

2. Eisner RF, Nieberg RK, Berek JS. Synchronous primary neoplasms of the female reproductive tract. Gynecologic oncology. 1989 Jun 1;33(3):335-9.

3. Zaino R, Whitney C, Brady MF, DeGeest K, Burger RA, Buller RE. Simultaneously detected endometrial and ovarian carcinomas - a prospective clinicopathologic study of 74 cases: a gynecologic oncology group study. Gynecologic oncology. 2001 Nov 1;83(2):355-62.

4. Scully REYR, Clement PB: Tumors of the Ovary, Maldeveloped Gonads, Fallopian Tube, and Broad Ligament: Atlas of Tumor Pathology. Bethesda, MD, Armed Forces Institute of Pathology, 1998

5. CHIANG YC, CHEN CA, HUANG CY, HSIEH CY, CHENG WF. Synchronous primary cancers of the endometrium and ovary. International Journal of Gynecological Cancer. 2008 Jan 1;18(1):159-64.

6. Lim YK, Padma R, Foo L, Chia YN, Yam P, Chia J, KhooTan HS, Yap SP, Yeo R. Survival outcome of women with synchronous cancers of endometrium and ovary: a 10 year retrospective cohort study. Journal of gynecologic oncology. 2011 Dec 31;22(4):239-43.

7. Liu Y, Li J, Jin H, Lu Y, Lu X. Clinicopathological characteristics of patients with synchronous primary endometrial and ovarian cancers: A review of 43 cases. Oncology letters. 2013 Jan 1;5(1):267-70.

8. van Niekerk CC, Bulten J, Vooijs GP, Verbeek AL. The association between primary endometrioid carcinoma of the ovary and synchronous malignancy of the endometrium. Obstetrics and gynecology international. 2009 Dec 15;2010.

9. Szmich SD, Czernek U, Krakowska M, Frąckowiak M, Zięba A, Czyżykowski R, Kulejewska D, Potemski P. 
Synchronous primary ovarian and endometrial cancers: a series of cases and a review of literature. PrzMenopauzalny. 2014 Mar;13:64-9.

10. Soliman PT, Slomovitz BM, Broaddus RR, Sun CC, Oh JC, Eifel PJ, Gershenson DM, Lu KH. Synchronous primary cancers of the endometrium and ovary: a single institution review of 84 cases. Gynecologic oncology. 2004 Aug 31;94(2):456-62.

11. Signorelli M, Fruscio R, Lissoni AA, Pirovano C, Perego $\mathrm{P}$, Mangioni C. Synchronous early-stage endometrial and ovarian cancer. International Journal of Gynecology\& Obstetrics. 2008 Jul 1;102(1):34-8.

*Corresponding author:

Rajendiran S, Department of pathology, Sri Ramachandra Medical college and Research Institute, Chennai (India)

Phone: +91 9480755187

Email: srajendiran@sriramachandra.edu.in,

Date of Submission : 24.01.2017

Date of Acceptance : 24.05.2017

Financial or other Competing Interests: None.

Date of Publication : 25.08.2017 